\title{
A hybrid time-domain model for pulsed terahertz dipole antennas
}

K. Ezdi

kamran.ezdi@ihf.tu-bs.de

B. Heinen

C. Jördens

N. Vieweg

R. Wilk

M. Mikulics

M. Koch
N. Krumbholz

Institut für Hochfrequenztechnik, Technische Universität Braunschweig, Schleinitzstraße 22, 38106 Braunschweig, Germany

Institut für Hochfrequenztechnik, Technische Universität Braunschweig, Schleinitzstraße 22, 38106 Braunschweig, Germany

Institut für Hochfrequenztechnik, Technische Universität Braunschweig, Schleinitzstraße 22, 38106 Braunschweig, Germany

Institut für Hochfrequenztechnik, Technische Universität Braunschweig, Schleinitzstraße 22, 38106 Braunschweig, Germany

Institut für Hochfrequenztechnik, Technische Universität Braunschweig, Schleinitzstraße 22, 38106 Braunschweig, Germany

Menlo Systems GmbH, Am Klopferspitz 19, 82152 Martinsried, Germany

Institut für Bio- und Nanosysteme, Forschungszentrum Jülich, Wilhelm-Johnen-Straße, 52428 Jülich, Germany

Institut für Hochfrequenztechnik, Technische Universität Braunschweig, Schleinitzstraße 22, 38106 Braunschweig, Germany

In this paper we study the effect of the length of dipole antennas on the spectrum of the radiated THz signal in pulse-excited opto-electronic terahertz systems. In particular, we investigate the origin of the commonly observed sharp dips that occur in the spectra of photoconductive dipole antennas, and explain them on the basis of reflections of the excitation current pulse that take place at the ends of the antenna. We develop a hybrid time-domain model for the system and show that the predictions of our model are in good agreement with experimental results. [DOI: 10.2971/jeos.2009.09001]

Keywords: pulse-excited antennas, time-domain model, THz time-domain spectroscopy, Drude-Lorentz model

\section{INTRODUCTION}

In recent years terahertz time-domain spectroscopy [1, 2] has gained popularity in basic research and in applications such as quality control [3]-[8] and national security [9]-[11]. The technique is based on the principle of optical excitation of a semiconductor substrate on which a metal structure is deposited. The optical pulse, usually a few tens of femtoseconds in duration, produces a transient photocurrent, which excites the antenna and produces pulses of $\mathrm{THz}$ radiation.

Most studies of pulse-excited terahertz systems focus on the short current pulse that is produced in the substrate as a result of the ultra-short optical excitation and treat this region as a point source for which the radiated electric field is directly proportional to the time-derivative of the current. This approach is correct in the first approximation but it ignores the influence of the propagation of the pulse on the antenna and the presence of the striplines and the contact pads on the spectrum of the radiated $\mathrm{THz}$ signal.

In this paper, we investigate the influence of the dipole structure on the radiated $\mathrm{THz}$ pulse by using Smith's model for pulse-excited antennas and explain the origin of the sharp dips that are often observed in experimental measurements, see e.g. [12]-[15]. In contrast with previous studies that consider the radiation as originating from a Hertzian dipole $[16,17]$, our analysis takes into account the more realistic case of the entire length of the antenna. We use the simple model for pulse-excited antennas described in detail by Smith $[18,19]$, and earlier developed by Franceschetti et al. in a pioneering paper [20]. We combine the results of this model with results previously derived by Jepsen et al. for the photocurrent produced by the ultrafast laser pulse excitation [21] and calculate the electric field after it has propagated through the emitter substrate and the hyper-hemispherical lens. To our knowledge, this is the first analytical approach towards calculating the electric field radiated from pulse-excited $\mathrm{THz}$ antennas that takes into account the length of the dipole by incorporating the effects of reflections that take place at its ends.

\section{THEORETICAL BACKGROUND}

In contrast with conventional antenna analysis, where the excitation is continuous-wave and time-harmonic, the analysis 
of antennas fed with short pulses is a less explored area, and consequently little material is available in the literature on this topic. This holds particularly true for our special case of terahertz antennas. Continuous-wave terahertz systems that operate on the principle of mixing of two laser beams of slightly different frequencies to produce terahertz radiation at the difference frequency, a process called photomixing, have been investigated in detail [22]-[24]. Brown et al. developed a circuit model for CW terahertz antennas that successfully predicts their behaviour [25]. However, no corresponding model has so far been developed for pulse-excited terahertz antennas.

We analyse the problem of pulse-excited terahertz antennas in the time domain, which is a more convenient approach, and which gives better physical insight compared to the frequency domain. Our starting point is the introduction of Smith's travelling-wave element, shown in Figure 1(a), which is the building block of the dipole antenna. The travelling-wave element is an ideal linear radiating structure with a signal source at one end and a perfect termination at the other. The source places the pulse on the element. The pulse travels down the length of the element and is absorbed by the termination. The termination is considered a perfect absorber in the sense that it does not reflect the signal back towards the source. The phys-

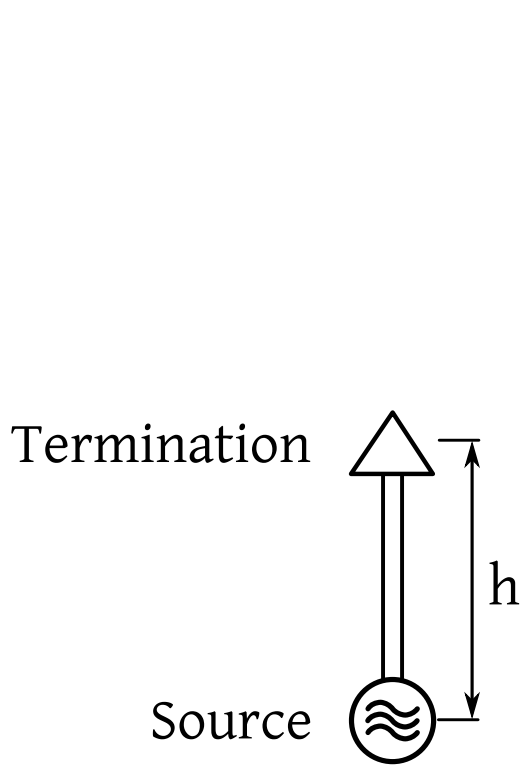

(a)
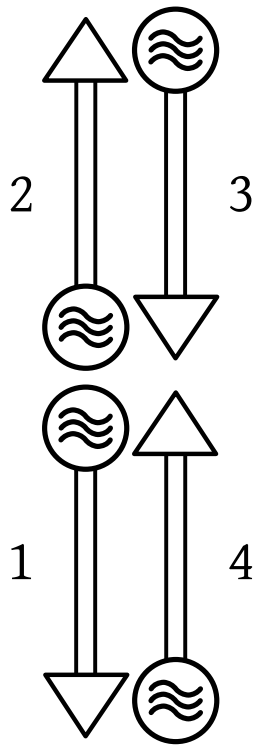

(b)
FIG. 1 Schematic diagram of (a) the basic travelling-wave element and (b) the dipole antenna made of four traveling-wave elements.

ical principle behind radiation is the acceleration of charge $[18,26]$. When the source places the pulse of current on the element, it results in an acceleration of charge, which produces radiation. If the travelling-wave element is assumed to be made of a perfect electric conductor, the pulse travels down the length of the element at a constant velocity and during this time it does not produce radiation. On reaching the termination, the pulse is absorbed, which causes a deceleration of charge, and consequently another pulse of radiation is generated.

A dipole antenna can be thought of as consisting of four travelling-wave elements joined together as shown in Figure 1(b). Elements 1 and 2 model the outgoing pulse of current while elements 3 and 4 are incorporated to take into account the reflection at the ends of the dipole. The wave completes one circuit by travelling one way up and one way down. In an ideal loss-less dipole, the process continues indefinitely with the pulse losing energy to radiation at every reflection.

\section{GEOMETRICAL SET-UP}

The schematic diagram of the radiating system is shown in Figure 2. The metallic structure on the semiconductor substrate consists of the contact pads, the striplines, and the dipole structure (shown in the dashed area), forming an $\mathrm{H}$ like structure. The ultrafast laser excitation is focussed on the photoconducting region between the two arms of the antenna. The contact pads provide connections to an external dc power source that supplies the bias field in the semiconductor region inside the antenna gap.

\section{Dipole structure}

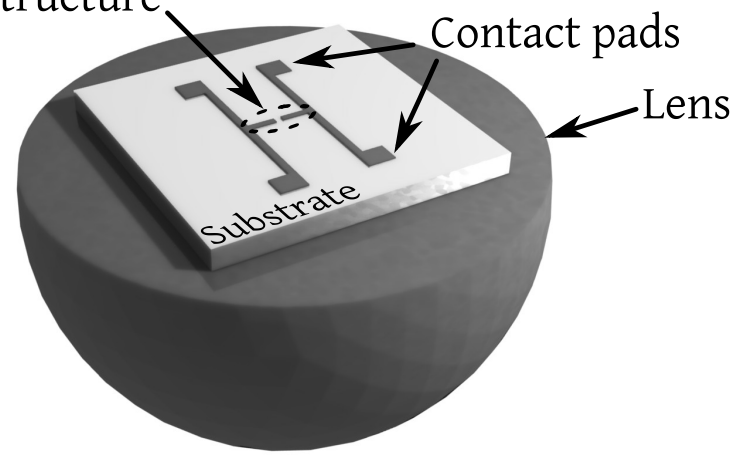

FIG. 2 Schematic diagram of a typical pulse-excited THz radiating system.

\section{THE MATHEMATICAL MODEL}

\subsection{The excitation photocurrent}

The ultrafast laser pulse produces electron-hole pairs in the semiconductor region between the arms of the antenna. These charge carriers are accelerated under the influence of the applied electric field, resulting in a short current pulse, which produces the pulsed $\mathrm{THz}$ radiation.

We use the modified Drude-Lorentz model, developed by Jepsen et al. [16] and later used by Piao et al. [27], to analyse the generation of the photocurrent. The process is described by the following set of three coupled partial differential equations relating the charge density $n_{f}$, the velocity of carriers $v$ and the polarisation caused by the screening of the bias field, $P_{S C}$ :

$$
\begin{aligned}
\frac{d n_{f}}{d t} & =-\frac{n_{f}}{\tau_{c}}+G(t) \\
\frac{d v}{d t} & =-\frac{v}{\tau_{s}}+\frac{e}{m^{*}} E_{l o c} \\
\frac{d P_{s c}}{d t} & =-\frac{P_{s c}}{\tau_{r}}+j(t)
\end{aligned}
$$


with

$$
E_{l o c}=E_{b i a s}-\frac{P_{s c}}{\eta \epsilon}
$$

and

$$
j(t)=-e \cdot n_{f} \cdot v,
$$

where $E_{b i a s}$ and $E_{l o c}$ represent the bias field and the local field resulting from the screening of the charge carriers, $\epsilon$ is the permittivity of the semiconductor substrate, $G(t)$ is the rate of generation of carriers, $\tau_{c}, \tau_{s}$ and $\tau_{r}$ are the carrier trapping time, the carrier scattering time and the carrier recombination time, respectively, $j(t)$ is the current density and $\eta$ is a geometrical factor. Using typical values available in the literature, these equations are solved numerically to get the photocurrent as a function of time. Figure 3 shows a plot of the calculated photocurrent.

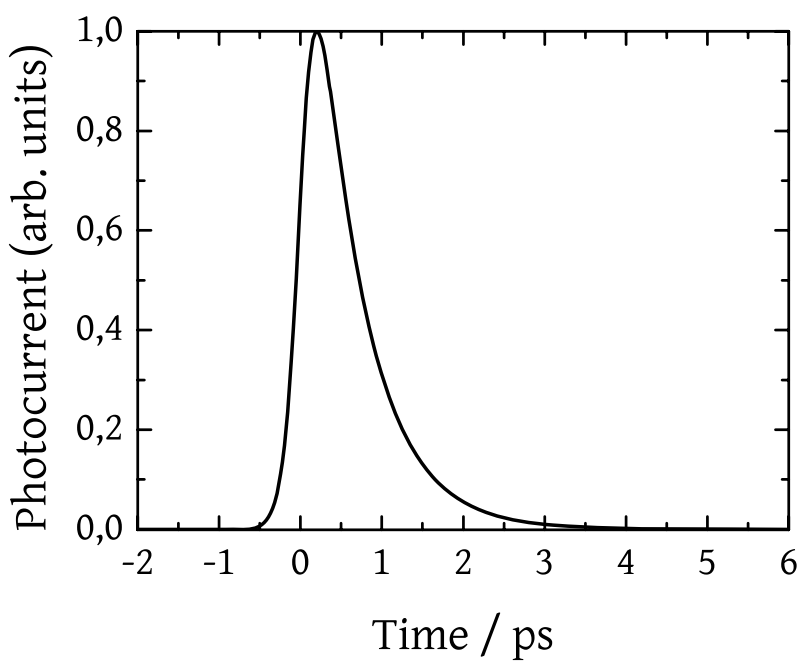

FIG. 3 Current pulse produced in the photoconductive area as a result of ultra-fast optical excitation.

\subsection{Incorporating the Smith model}

Having calculated the photocurrent, the modified Smith model is now incorporated in the analysis. In the simple, free-space Smith model, the radiated electric field, in the far-field region, is given by:

$$
\begin{aligned}
E_{R}(r, t)=\frac{\mu_{0} c}{2 \pi r \sin \left(\theta_{A n t}\right)} & \left\{I_{S}\left(t-\frac{r}{c}\right)\right. \\
& +I_{S}\left(t-\frac{r}{c}-\frac{2 \cdot h}{c}\right) \\
& -I_{S}\left(t-\frac{r}{c}-\frac{h}{c} \cdot\left(1-\cos \left(\theta_{A n t}\right)\right)\right) \\
& \left.-I_{S}\left(t-\frac{r}{c}-\frac{h}{c} \cdot\left(1+\cos \left(\theta_{\text {Ant }}\right)\right)\right)\right\}
\end{aligned}
$$

where $I_{S}$ is the excitation current, $r$ is the distance to the field point, $h$ is the length of one arm of the dipole, $c$ is the velocity of light and $\theta_{A n t}$ is the elevation angle of the spherical coordinate system. The argument of the current shows that the electric field at the field point is caused by the current at the retarded time $\left(t-\frac{h}{c}\right)$. Note that the radiated electric field is proportional to the excitation current, not its time-derivative. This seemingly surprising result is a direct consequence of solving Maxwell's equations subject to the geometrical boundary conditions of the dipole, and is not in contradiction with them. The detailed mathematical derivation of this result is shown in $[18,28]$.

To adapt the Smith model to the terahertz antenna, the presence of the substrate is accounted for by modifying the velocities in the above expression. The velocity of propagation along a conductor with a dielectric on one side and free space on the other $\left(v_{1}\right)$ is given by [17]:

$$
v_{1} \approx \frac{c}{\sqrt{\epsilon_{e f f}}}
$$

where

$$
\epsilon_{e f f}=\frac{\epsilon_{r}+1}{2},
$$

while the velocity of propagation in the substrate $\left(v_{2}\right)$ is given by

$$
v_{2}=\frac{c}{\sqrt{\epsilon_{r}}} .
$$

The value of the wave-propagation velocity for a GaAs substrate, as given by the commonly-used formula in Eq. (7), is $1.14 \times 10^{8} \mathrm{~ms}^{-1}$. As the cross-sectional area of our antenna is not infinitesimally small, the use of this equation can lead to erroneous results. To avoid this, we use a commercially available simulation program [29] to numerically obtain the velocity of propagation on the dipole. In our analysis we use an average value of $0.96 \times 10^{8} \mathrm{~ms}^{-1}$, obtained from several different simulations, rather than the one predicted by the approximation in Eq. (7).

Incorporating these velocities and the photocurrent derived above gives the modified expression for the radiated electric field:

$$
\begin{aligned}
E_{R}(r, t)=\frac{\mu_{0} c}{2 \pi r \sin \left(\theta_{A n t}\right)} & \left\{I_{S}\left(t-\frac{r}{v_{2}}\right)\right. \\
& \left.-I_{S}\left(t-\frac{r}{v_{2}}-\frac{2 \cdot h}{v_{1}}\right)\right\}
\end{aligned}
$$

Here, the first term denotes the field radiated by the pulse of current as it is launched on the dipole (the acceleration term) while the second term denotes the electric field produced as the reflected current pulse reaches the source back (the deceleration term). Note that the radiation produced at the ends of the antenna, represented by the third and fourth terms in Eq. (6), has not been included in this formulation. This is because the radiation from the ends is not collimated by the hyper-hemispherical lens, and is, therefore, not picked up by the detector [16]. The two factors contributing to the radiation in this model are the original excitation pulse and its return back to the centre of the dipole. The change in sign for the deceleration term is explained by noting that in Smith's model the return pulse is completely absorbed whereas in our particular case, the pulse is reflected. Note further that if the dipole were treated as a Hertzian dipole, as in previous studies, there would be no reflected pulse. 
We make certain simplifying assumptions in the analysis. Firstly, we neglect the attenuation of the current pulse, especially at high frequencies, that would make it lose energy continuously as it moves. Secondly, we take the reflection coefficient at the ends of the dipole to be exactly unity. However, as we show later, these assumptions do not qualitatively change the predictions of our model, as supported by the reasonably good experimental evidence that we present.

\subsection{The influence of the lens and the detector}

The field produced by the antenna (Eq. (10)) propagates through the dielectric substrate and the hyper-hemispherical lens attached to it. We use the time-domain Fresnel-Huygens diffraction integral [30] in conjunction with the field radiated by the antenna $E_{R}$ :

$$
E_{Q} \approx-\frac{1}{4 \pi} \frac{\partial}{\partial t} \int_{S} \frac{E_{R}\left(t-\frac{r^{\prime}}{c}\right)}{r^{\prime}}\left(\cos \left(\mathbf{n}_{\mathbf{s}}, \mathbf{r}^{\prime}\right)-\mathbf{n}_{\mathbf{1}} \cos \left(\mathbf{n}_{\mathbf{s}}, \mathbf{r}\right)\right) d S
$$

and integrate numerically over the lens surface, excluding the part of the surface where the radiation is reflected back due to total internal reflection (Figure 4). This gives the electric field $E_{Q}$ at a point outside the lens, after it has travelled through the lens. After propagating through the lens and the interven-

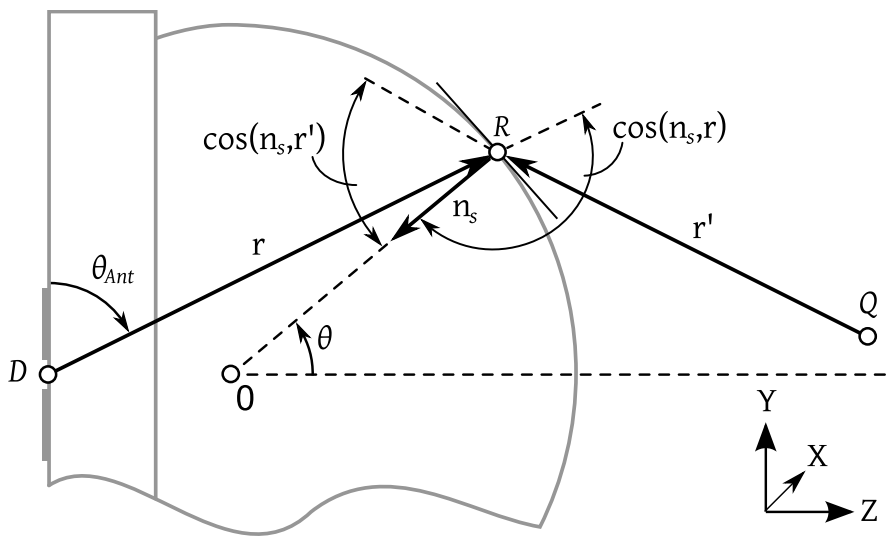

FIG. 4 Schematic diagram of propagation through the hyper-hemispherical lens and the solution of the Fresnel-Kirchhoff diffraction integral.

ing space, the radiated terahertz field encounters the detector where it causes a current flow. Ideally, the detector should faithfully reproduce the incident electric field without any distortion. However, all real detectors modify the incident field signal to some extent, with the result that the detector current is not an exact replica of the radiated field. In our analysis we use the detector transfer function developed by Jepsen et al. [16] that relates the incident terahertz electric field to the detector current. Note that the geometrical complexity of pulseexcited THz systems, and their different method of reception compared to conventional transmit-receive systems, preclude a direct application of the time-domain reciprocity theorem. This prevents the development of a similar model for the detector antenna. However, as the phenomenon of pulse propagation is also present on the detector antenna, the predictions of our model are also applicable to the detector antenna.

\section{RESULTS}

\subsection{The received time-domain signal}

In Figure 5 we show the calculated time-domain plots of the detector signal for two different antenna lengths predicted by our hybrid model. Two important features can be observed. Firstly, it can be seen that the detector current is proportional to the second derivative of the excitation current, the two time-differentiations coming from the two hyperhemispherical lenses at the emitter and the detector. This result is consistent with both the modified Smith formula (Eq. (10)) and the time-domain form of the Fresnel-Kirchhoff diffraction integral (Eq. (11)). Eq. (10) shows that the radiated electric field is proportional to the excitation current and Eq. (11) shows that the time-differentiation originates from propagation through the hyper-hemispherical lens. Note that this approach differs from previous analyses [16, 17] where the differentiation is identified as coming from the antenna.

Secondly, the effect of the reflected current pulse can also be clearly seen from the two plots. The influence of the reflected pulse on the radiated signal is more pronounced in the case of the longer antenna. For the shorter antenna the reflected pulse smears into the main pulse.

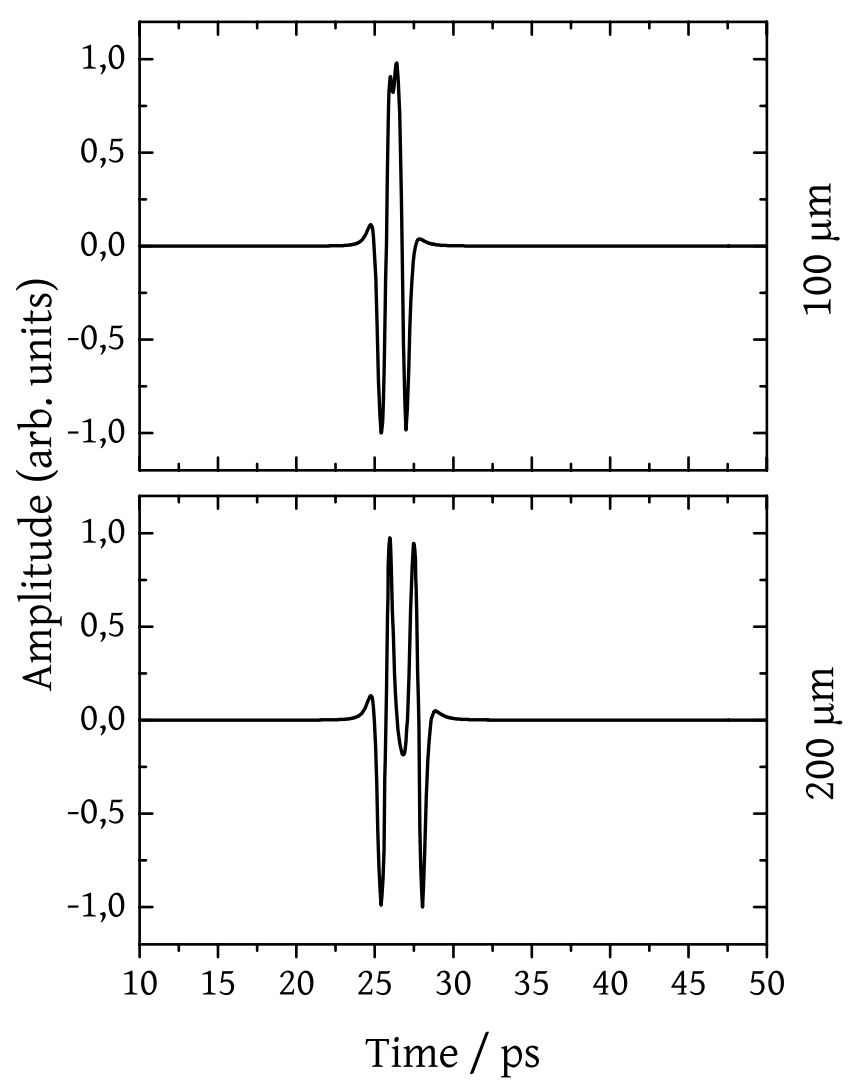

FIG. 5 Simulation results for the received time-domain signal for two dipole lengths of the emitter.

\subsection{Experimental verification}

To confirm the predictions of our model, we carried out several experiments on different pulse-excited systems with varying dipole lengths. The antennas were fabricated on differ- 
ent materials. The comparison between theoretical and experimental results is shown in Figures $6-8$.

Figure 6 shows experimental and simulation results for the case of a $150 \mu \mathrm{m}$ emitter dipole and a $20 \mu \mathrm{m}$ detector dipole deposited on different substrates. An illustrative time-domain

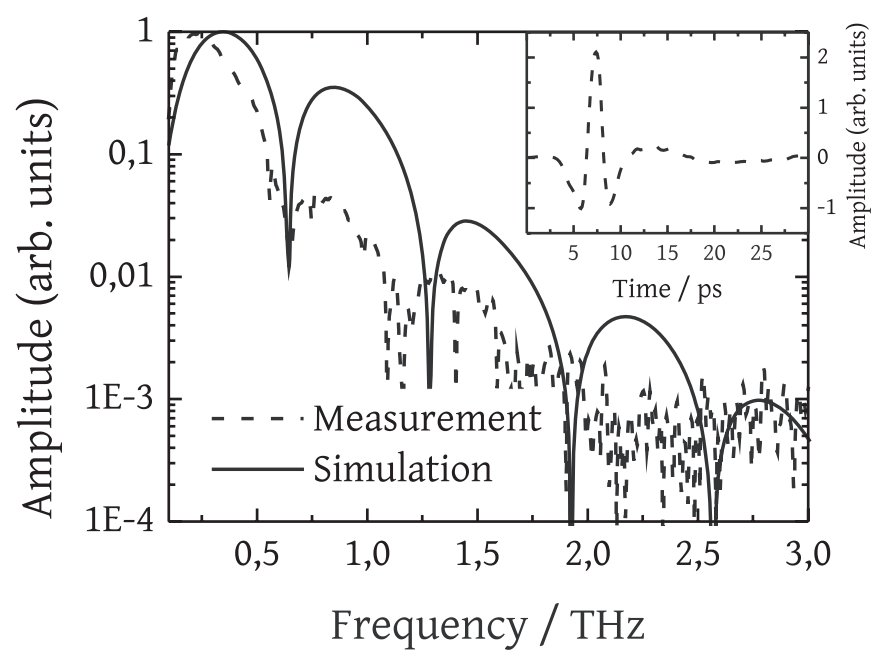

FIC. 6 Experimental and simulation results for a $150 \mu \mathrm{m}$ emitter antenna on InGaAs with a $5 \mu \mathrm{m}$ gap and a $20 \mu \mathrm{m}$ detector antenna on Silicon-on Sapphire with a $15 \mu \mathrm{m}$ gap.

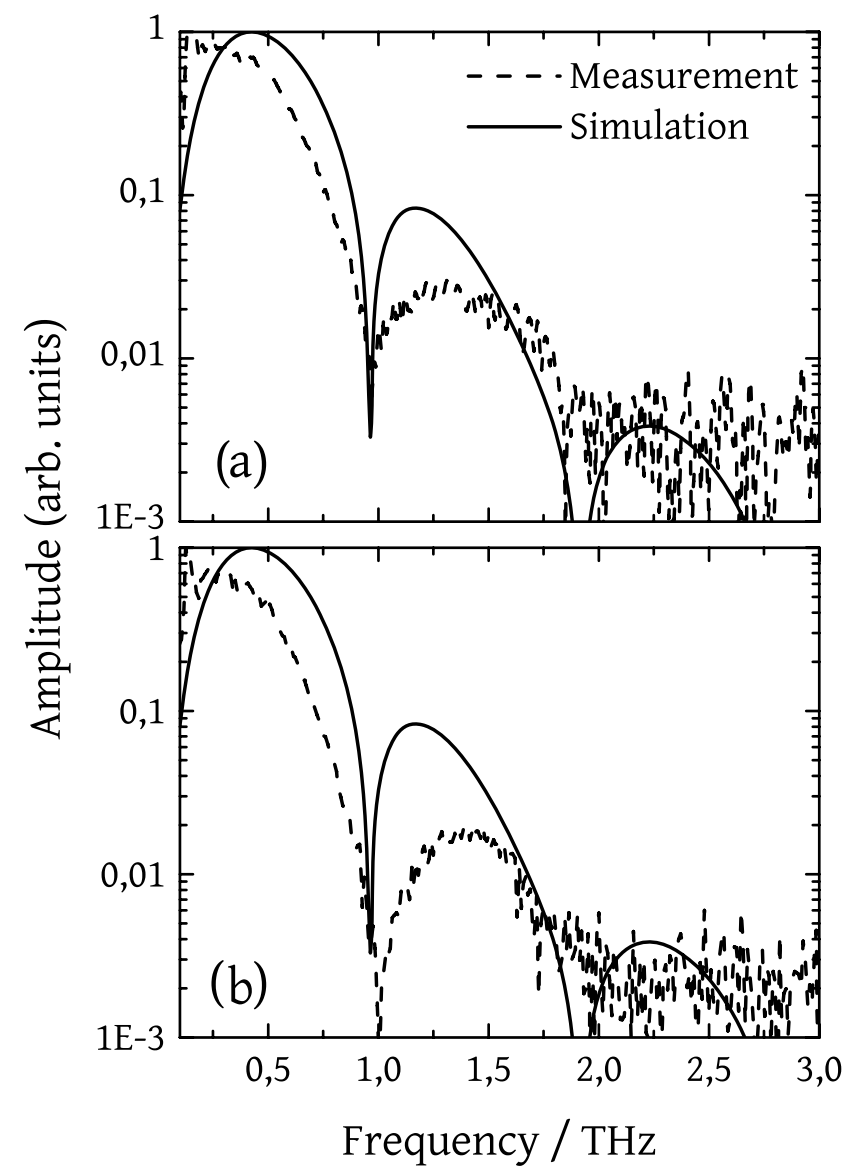

FIG. 7 Experimental and simulation results for (a) stripline emitter antenna on GaAs with a $50 \mu \mathrm{m}$ separation and $100 \mu \mathrm{m}$ detector antenna on InGaAs with a $15 \mu \mathrm{m}$ gap and (b) $100 \mu \mathrm{m}$ emitter and detector antennas on InGaAs with $15 \mu \mathrm{m}$ gaps. plot of the measured signal is also shown as an inset. Owing to its very short length, the detector dipole does not have a significant influence on the spectrum in the frequency range considered here. Its effect occurs at frequencies above $3 \mathrm{THz}$, and therefore the main features of the spectrum, including the dips, can be attributed to the emitter only. Note that this corresponds very well to the requirements of our model. From the figure a good degree of agreement between the predictions of the model and experimental results can be seen, especially at low frequencies. One possible reason for the discrepancy observed at higher frequencies is the dispersion of the signal as it propagates on the dipole. Our model, being formulated in the time domain, does not include the effects of dispersion.

The results in Figure 7(a) were obtained with a simple stripline emitter and a $100 \mu \mathrm{m}$ dipole detector with a $15 \mu \mathrm{m}$ photoconductive gap in the middle. The stripline emitter is a simple structure consisting of two parallel metal electrodes deposited on a semiconductor substrate. The emitter and detector were deposited on GaAs and InGaAs, respectively. It is pointed out that for this case, the theoretical results were obtained by making the assumption that the emitter is a $100 \mu \mathrm{m}$ dipole and the receiver is an ideal detector. In other words, the positions of the emitter and receiver are inverted in the theoretical model. From the plot it can be seen that despite the inversion, the results of the theory agree well with the experiment. The dips in this case come from the detector dipole, leading to the conclusion that the predictions of our model are also applicable to the detector. This is so because the phenomenon of pulse propagation is also present at the detector. The onset of noise at frequencies higher than $2 \mathrm{THz}$ in the experimental results prevents an exact comparison at higher frequencies. However, a general trend towards agreement is clearly visible.

In Figure 7(b) the results for identical-length emitter and detector dipoles on InGaAs are shown. The only difference from the case of Figure 7(a) is that the stripline emitter has been replaced with a dipole antenna. Like in the previous case, the experimental results agree well with the predictions of the theory. In particular, it can be seen that although the position of

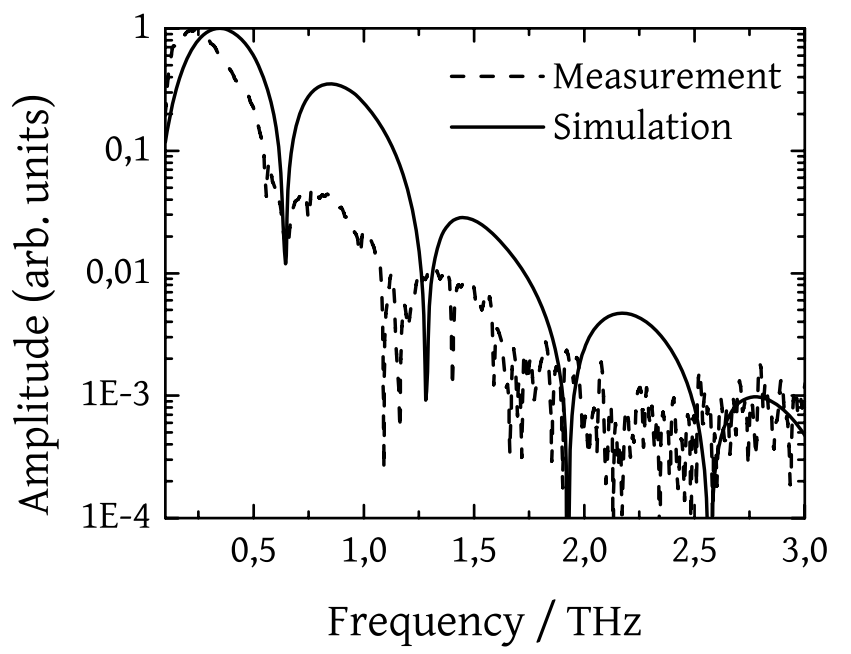

FIG. 8 Experimental and simulation results for (a) $100 \mu \mathrm{m}$ emitter and detector antennas on LT-GaAs and (b) $200 \mu \mathrm{m}$ emitter and detector antennas on LT-GaAs. 
the dip is only slightly shifted, its width is broadened. This supports our earlier assertion that our model is also applicable to the detector antenna.

Figures 8(a) and 8(b) show the comparison for the case of fibre-coupled antennas having identical dipole lengths of emitter and detector. The antennas were fabricated on LTGaAs and had lengths of $100 \mu \mathrm{m}$ and $200 \mu \mathrm{m}$ respectively and were excited with fibre-guided femtosecond laser pulses. Again, the theoretically predicted dips coincide with the experimental results, particularly at low frequencies, to within a few percent. However, the agreement between experiment and theory becomes less convincing at high frequencies. As mentioned earlier, one possible reason for this behaviour is the dispersion of the signal as it propagates on the dipole.

\section{DISCUSSION AND CONCLUSION}

As we mentioned in the introduction, most analyses of pulseexcited terahertz antennas reported in the literature limit attention to the active photoconductive area between the metal electrodes. For most commonly-used structure geometries, the physical dimensions of this area are much smaller than the significant wavelengths in the current pulse. Consequently, this area is treated as a Hertzian dipole for which the radiated electric field is proportional to the first derivative of the excitation current.

This approach, however, neglects the influence of the propagation of the current pulse on the finite-length metal structure (the H-structure) whose length cannot be neglected in comparison with the wavelengths involved. Our analysis goes further than these studies by incorporating the entire length of the metal structure instead of the photoconductive gap alone. We treat the pulse-excited $\mathrm{THz}$ emitter as a finite-length dipole instead of as a Hertzian dipole. In our opinion, this is a physically more realistic approach as it explains the origin of the commonly observed dips in the measured spectra on the basis of the superposition of the incident excitation current pulse and the time-delayed pulse that is reflected from the ends of the dipole. Additionally, it can be deduced from the measurements that this phenomenon of pulse propagation on the finite-length dipole also takes place at the detector, causing dips in a similar manner as for the emitter. The predictions of our model are supported reasonably well by various experimental results.

Our model also highlights the important but less known fact that the time-differentiation of the excitation current is caused by propagation through the hyper-hemispherical lens and not by the antenna itself. As explained above, this is due to the fact that we consider the antenna a finite-length dipole, not a Hertzian dipole, for which the radiated field is proportional to the excitation current, not its time-derivative. This fact is derived mathematically in $[18,28]$.

In conclusion, we have adapted the Smith model for pulseexcited dipole antennas to the special case of pulse-excited terahertz antennas. Despite the simplicity of our model, and the approximations involved, the results predicted by it, in particular the origin and position of the dips in the measured spectrum, agree well with experimental results.

\section{References}

[1] L. Duvillaret, F. Garet, and J. L. Coutaz, "Influence of noise on the characterization of materials by terahertz time-domain spectroscopy" J. Opt. Soc. Am. B 17, 452-461 (2000).

[2] M. Tonouchi, "Cutting-edge terahertz technology" Nat. Photonics 1, 97-105 (2007).

[3] S. Hunsche, D. M. Mittleman, M. Koch, and M. C. Nuss, "New dimensions in T-ray imaging" IEICE T. Electron. E81-C, 269-275 (1998).

[4] M. Brucherseifer, P. Haring Bolivar, H. Klingenberg, and H. Kurz, "Angle-dependent THz tomography - Characterization of thin ceramic oxide films for fuel cell applications" Appl. Phys. B-Lasers 0. 72, 361-366 (2001).

[5] S. Wietzke, C. Jansen, F. Rutz, D. M. Mittleman, and M. Koch, “Determination of additive content in polymeric compounds with terahertz time-domain spectroscopy" Polym. Test. 26, 614-618 (2007).

[6] W. L. Chan, K. Charan, D. Takhar, K. F. Kelly, R. G. Baraniuk, and D. M. Mittleman, "A single-pixel terahertz imaging system based on compressed sensing" Appl. Phys. Lett. 93, 121105 (2008).

[7] C. Jördens and M. Koch, "Detection of foreign bodies in chocolate with pulsed THz spectroscopy" Opt. Eng. 47, 037003 (2008).

[8] N. Krumbholz, T. Hochrein, N. Vieweg, T. Hasek, K. Kretschmer, M. Bastian, M. Mikulics, and M. Koch, "Monitoring polymeric compounding processes inline with $\mathrm{THz}$ timedomain spectroscopy" Polym. Test. in press, (2008) (doi:10.1016/j.polymertesting.2008.09.009).

[9] J. F. Federici, B. Schulkin, F. Huang, D. Gary, R. Barat, F. Oliveira, and D. Zimdars, "THz imaging and sensing for security applications - Explosives, weapons and drugs" Semicond. Sci. Tech. 20, S266S280 (2005).

[10] H. B. Liu, Y. Chen, G. J. Bastiaans, and X. Zhang, "Detection and identification of explosive RDX by THz diffuse reflection spectroscopy" Opt. Express 14, 415-423 (2006).

[11] P. U. Jepsen, J. K. Jensen, and U. Müller, “Characterization of aqueous alcohol solutions in bottles with THz reflection spectroscopy" Opt. Express 16, 9318-9331 (2008).

[12] R. Buhleier, Elektrodynamische Eigenschaften von Hochtemperatur-Supraleitern im THz-Bereich, Ph.D. thesis, (Max-Planck-Institut für Festkörperforschung, Stuttgart, 1994).

[13] Y. Cai, I. Brener, J. Lopata, J. Wynn, L. Pfeiffer, J. B. Stark, Q. Wu, X. C. Zhang, and J. F. Federici, "Coherent terahertz radiation detection: Direct comparison between free-space electro-optic sampling and antenna detection" Appl. Phys. Lett. 73, 444-446 (1998).

[14] R. Yano, H. Gotoh, Y. Hirayama, S. Miyashita, Y. Kadoya, and T. Hattori, "Terahertz wave detection performance of photoconductive antennas: Role of antenna structure and gate pulse intensity" Jpn. J. Appl. Phys. 97, 1-6 (2005).

[15] R. Wilk, Switchable THz Relectors (Cuvillier Verlag, Göttingen, 2007).

[16] P. U. Jepsen, R. H. Jacobsen, and S. R. Keiding, "Generation and detection of terahertz pulses from biased semiconductor antennas" J. Opt. Soc. Am. B 13, 2424-2436 (1996).

[17] M. Tani, S. Matsuura, K. Sakai, and S. Nakashima, "Emission characteristics of photoconductive antennas based on lowtemperature-grown GaAs and semi-insulating GaAs" Appl. Optics. 


\section{6, 7853-7857 (1997).}

[18] G. Smith, An Introduction to Classical Electromagnetic Radiation (Cambridge University Press, 1997).

[19] G. S. Smith, "Teaching antenna radiation from a time-domain perspective" Am. J. Phys. 69, 288-300 (2001).

[20] G. Franceschetti and C. Papas, "Pulsed antennas" IEEE T. Antenn. Propag. 22, 651-661 (1974).

[21] P. U. Jepsen, Generation and applications of THz radiation, Ph.D. thesis (Aarhus University, Denmark, 1996).

[22] S. Matsuura, M. Tani, and K. Sakai, "Ceneration of coherent terahertz radiation by photomixing in dipole photoconductive antennas" Appl. Phys. Lett. 70, 559-561 (1997).

[23] S. M. Duffy, S. Verghese, K. A. McIntosh, A. Jackson, A. C. Cossard, and S. Matsuura, "Accurate modeling of dual dipole and slot elements used with photomixers for coherent terahertz output power" IEEE T. Microw. Theory. 49, 1032-1038 (2001).

[24] I. S. Gregory, W. R. Tribe, B. E. Cole, M. J. Evans, E. H. Lin- field, A. G. Davies, and M. Missous, "Resonant dipole antennas for continuous-wave terahertz photomixers" Appl. Phys. Lett. 85, 1622-1624 (2004).

[25] E. R. Brown, F. W. Smith, and K. A. McIntosh, "Coherent millimeterwave generation by heterodyne conversion in low-temperaturegrown GaAs photoconductors" Jpn. J. Appl. Phys. 73, 1480-1484 (1993).

[26] J. D. Jackson, Classical Electrodynamics, 3rd edn. (Wiley, 1998).

[27] Z. Piao, M. Tani, and K. Sakai, "Carrier dynamics and terahertz radiation in photoconductive antennas" Jpn. J. Appl. Phys. 39, 96100 (2000).

[28] M. A. Uman, D. K. McLain, and E. P. Krider, "The electromagnetic radiation from a finite antenna" Am. J. Phys. 43, 33 (1975).

[29] Microwave Studio 2008 (CST - Computer Stimulation Technology, 2008).

[30] M. Born and E. Wolf, Principles of optics (Cambridge University Press, 1980). 